\title{
Rotation and Properties of Be Stars
}

\author{
J. Zorec \\ Institut d'Astrophysique de Paris, UMR7095/CNRS, 98 bis bd. Arago, \\ F-75014 Paris, France (zorec@iap.fr)
}

\begin{abstract}
The actual characteristic average surface angular velocity ratio of the Be phenomenon may be higher than $\Omega / \Omega_{\mathrm{c}}=0.8 \pm 0.1$ derived from $v \sin i$ values not corrected from gravity darkening effects. Late Be stars need not only higher values of $\Omega / \Omega_{\mathrm{c}}$, but also larger main sequence age ratios $\tau / \tau_{\mathrm{MS}}$ than early Be stars to display the Be phenomenon. Galactic field Be stars appear at any MS phase, but their frequency is higher for $\tau / \tau_{\mathrm{MS}} \gtrsim 0.5$. Be stars are probably neat differential rotators, i.e. they rotate with energy ratios kinetic/|gravitational| larger than withstood by critical rigid rotators. Initial formation conditions must favor the existence of fast rotators, while only a small fraction of them could be accounted for by mass transfer in close binary systems. Bn stars could perhaps represent a pre-Be phase.
\end{abstract}

\section{Introduction}

Dwarf to giant B stars having shown some emission in the Balmer lines at least once are called "classical" Be stars (Jaschek et al. 1981). The Be phenomenon also concerns late $\mathrm{O}$ and early A type stars (Marlborough 2000). Depending on the spectroscopic aspect of emissions and absorptions produced by the circumstellar envelope (CE) in the Balmer lines, a given Be star is called B-normal, Be, Be-shell or B-shell (Hubert \& Hubert 1979). As a Be star can show any of these aspects at a given moment, also called "phases of Be stars", they do not identify physically different objects, but changing structures and/or ionization states of the $\mathrm{CE}$. Phase variations are also seen in the visible continuum spectrum (Moujtahid et al. 1998, 1999). Be stars also display variable spectroscopic, spectrophotometric, polarimetric and interferometric characteristics over the whole spectrum that reveal, on the one hand, activities in the outermost stellar layers that are probably related to the formation of the CE and, on the other hand, describe the structure of the CE itself (general references in Slettebak 1976, Jaschek \& Groth 1982, Slettebak \& Snow 1987, Balona et al. 1994, Smith et al. 2000, Yudin 2001). Also probably relevant to the formation of the CE are the non-radial pulsations (NRP) of Be stars (Rivinius et al. 1998) and the magnetic fields (Neiner 2002, Neiner et al. 2002). According to their distribution in our Galaxy, kinematics and occurrence in binaries, Be stars seem to have roughly the same ages and apparently the same origin as B stars of same spectral type (Abt 1987, Zorec 1998). Depending on where they are found, Galactic field or clusters, or extragalactic, the Be phenomenon concerns from $17 \%$ to nearly $50 \%$ of non-supergiant B stars (Zorec \& Briot 1997, Maeder 

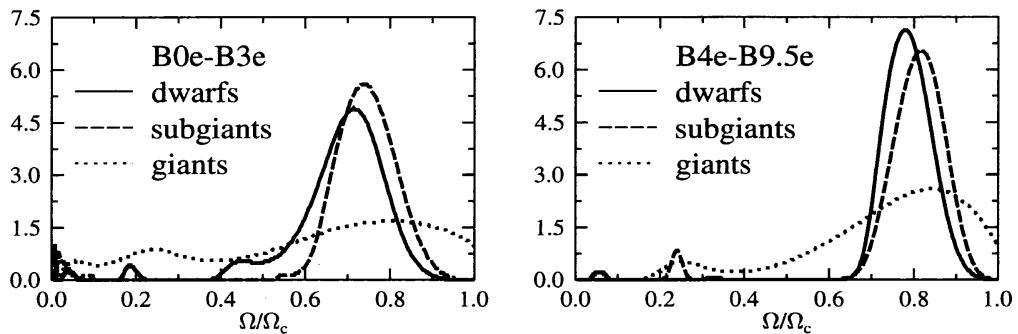

Figure 1. Probability distribution of the Be star angular velocity ratio $\omega=$ $\Omega / \Omega_{\mathrm{c}}$ for several spectral type- and luminosity class-groups

et al. 1999). The Be "character" is statistically stronger the hotter the spectral type and the larger the $v \sin i$ (Briot 1986).

The fundamental questions raised by the Be phenomenon concern the origin and/or the evolutionary status of stars that underly the CE and how the CE are formed. Excepting rare cases, fast rotation is one of the most outstanding characteristics of Be stars. The Be phenomenon can be thought of intrinsic or extrinsic nature. Thus, it has been assumed to be due either by a critical rotation at the secondary contraction phase, or by innate properties that come into play during the stellar main sequence (MS) evolution. It could also be an "acquired" phenomenon caused by interactions in binary systems.

\section{Be Stars as Rapid Rotators}

The first model of Be stars was proposed by Struve (1931, 1942). As B stars are centrally condensed objects with average polytropic index $n>0.81$, he assumed that a Be star could be a secularly stable (non-Jacobian ellipsoid) B star at rigid critical rotation (Tassoul 1978), which by equatorial mass ejection can produce an outer rotating extended envelope. On average, Be stars rotate faster than B stars without emission. Wolf \& Preston (1978) have shown that B6e-B9e stars rotate marginally faster than $\mathrm{B} 0 \mathrm{e}-\mathrm{B} 5 \mathrm{e}$ stars. Assuming that the distributions of true rotational velocities $(v \sin i$-distributions corrected statistically from the $\sin i$ factor) can be parametrized with a single parameter $p$ that represents their width, Fukuda (1982) showed that $p(\mathrm{~B})>p(\mathrm{Be})$ and that $p(\mathrm{~B} 0 \mathrm{e}-\mathrm{B} 2 \mathrm{e})>0.3$, while $p$ (B3e-B9e) $\lesssim 0.3$. Recently, Yudin (2001) showed that $0.38 \lesssim v \sin i / V_{\mathrm{c}} \lesssim 0.65\left(v_{\mathrm{c}}\right.$ $=$ critical equatorial velocity), so that $0.55 \lesssim v / V_{\mathrm{c}} \lesssim 0.65$, where the ratios are larger the later the spectral type groups and the more evolved the luminosity classes. Using newly determined $v \sin i$ parameters for a large sample of Be stars, we have shown (this volume, p. 85) that the true rotational velocities $v$ of Be stars are larger than those of $B$ stars by factors ranging from 1.5 to 2.5 . These authors also show that the increase of the true velocities $v$ among late type $B$ stars is more pronounced than for late Be stars and that the separation of the $v$ curves against spectral type of different luminosity class groups is higher for B than for Be stars. This can be the consequence of rotational effects on the spectral classification of Be stars, which make them appear more evolved than they actually are. The frequency distributions of $v$ for $\mathrm{B}$ stars are wide and irregular, due perhaps to the presence of stars with different physical characteristics ( $\beta \mathrm{Cep}, \mathrm{Bn}, \mathrm{Bp}, \mathrm{B})$, while those of $\mathrm{Be}$ stars are sharply peaked around specific values of $v$. Fig. 

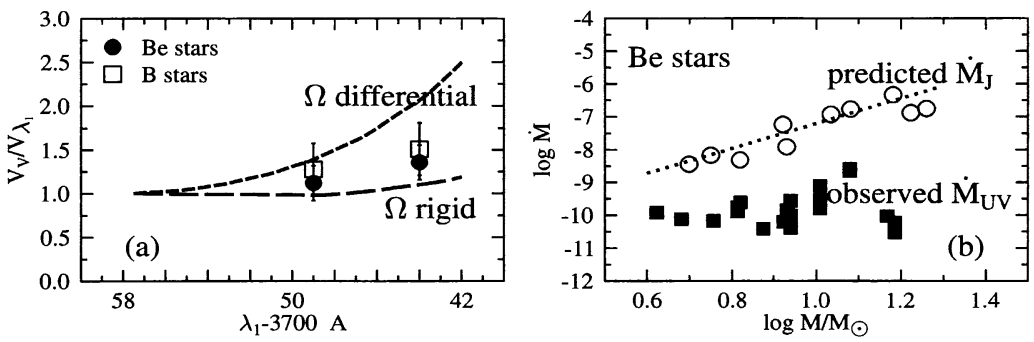

Figure 2. (a) Differential vs. rigid rotation of $\mathrm{B}$ and Be stars. $v_{\mathrm{V}} / v_{\lambda_{1}}=$ ratio between the true rotational velocity of dwarfs and that in other luminosity class groups; (b) predicted $\dot{M}_{\mathrm{J}}$ vs. observed $\dot{M}_{\mathrm{UV}}$ mass-loss rates

1 shows the distributions of the angular velocity ratio $\omega=\Omega / \Omega_{\mathrm{c}}\left(\Omega_{\mathrm{c}}=\right.$ critical angular velocity) of $\mathrm{Be}$ stars of different spectral types and luminosity class groups. The $\omega$ curves of giant Be stars are widespead, because many stars may have rotationally modified luminosity classes. In the remaining cases, Be stars seem to have $\omega=0.8 \pm 0.1$. Moreover, the more luminous and later the spectral types, the faster the stars need to rotate in order to display the Be phenomenon. We note however, that while the $v \sin i$ used to obtain the $\omega$ distributions in Fig. 1 are free from limb-darkening induced errors (Collins \& Truax 1995, Chauville et al. 2001), they can still be underestimated due to the "saturation" of spectral line widths carried at $\omega \gtrsim 0.8$ by the gravity darkening effect, which was not taken into account explicitly (Stoeckley 1968b, Howarth 2004, Owocki 2004). Correction for this effect might imply $0.8 \lesssim \omega \lesssim 1.0$.

\section{Internal Rotation of Be Stars}

The characteristics of stellar internal rotation are determined by the instabilities of several kinds, mass loss and the stellar evolution (Endal \& Sofia 1979, Zahn 1992, Maeder \& Zahn 1998). Be stars not only present a continuing and variable mass loss through winds which depends little or not al all on their surface rotation (Vardya 1985), but they also undergo sporadic massive ejections $\left(\Delta M \sim 10^{-11}-10^{-9} \mathrm{M}_{\odot}\right.$ : Hanuschik et al. 1993, Floquet et al. 2000, Hubert et al. 2000 , Zorec et al. 2000a,b) where the most intense ejections can be associated with the short- or long-lived light outbursts (Cook et al. 1995, Hubert \& Floquet 1998, Mennickent et al. 2002). Differences can then be expected between the evolution of the surface rotational velocities of B and Be stars. They may also imply distinctive behaviours of their respective internal rotations. Fig. 2a shows the surface velocity ratio $v_{\mathrm{V}} / v_{\lambda_{1}}$ as a function of the BCD luminosity class parameter $\lambda_{1}\left[v_{\mathrm{V}}=\right.$ equatorial velocity of dwarfs; $\overline{\lambda_{1}-3700}=57 \AA$ (dwarfs), 49 $\AA$ (subgiants), $44 \AA$ (giants)] calculated for two extreme cases: rigid and differential rotation (respectively with and without radial exchange of angular momentum). The inertial momentum of stars was determined from models of rotating stars calculated using Clement's (1974) algorithm with barotropic relations $P=P(\rho)$ of non-rotating models with $Z=0.02$ at several evolutionary phases (Schaller et al. 1992). The velocity ratios $v_{\mathrm{V}} / v_{\lambda_{1}}$ for $\mathrm{B}$ and $\mathrm{Be}$ stars were estimated by taking into account spectral type changes of stars due to rotation and evolution. Fig. 2a suggests then that neither B nor Be stars evolve as rigid rotators. 

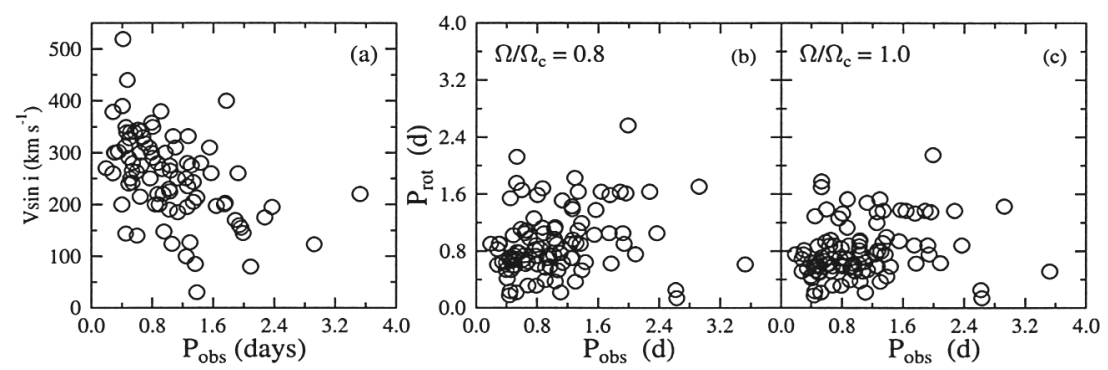

Figure 3. (a) $v \sin i$ vs. photometric pulsational periods $\mathrm{P}_{\text {obs }}$ of Be stars; (b) rotational periods $\mathrm{P}_{\text {rot }}$ for $\omega=0.8$ vs. observed pulsational periods and the same in (c) for $\omega=1.0$

Moreover, Be stars seem to have a weaker internal differential rotation than $\mathrm{Be}$ stars. This could be a spurious effect introduced by the luminosity classes of Be stars, which are probably not genuine stellar evolution idicators. Previous indications for differential rotation in B and Be stars were obtained by Balona (1975), Stoeckley (1968a) and Stoeckley and Buscombe (1987).

Assuming rigid rotation and using the same models as above, we calculated the differences of angular momenta of dwarfs and giant Be stars and interpreted them in terms of mass-loss rates $\dot{M}_{\mathrm{J}}$. Fig. $2 \mathrm{~b}$ shows the inferred $\dot{M}_{\mathrm{J}}$ rates compared with the $\dot{M}_{\mathrm{UV}}$ mass-loss rates derived from observed far-UV lines of Be stars (Snow 1981). Both series of points must still be brought closer by $\Delta \dot{M} \sim 0.4$ dex to account for the discrete mass ejections and the longer time spent by rotating stars in the MS. In spite of these corrections, both mass-loss rate estimates still differ by factors as high as $10^{2}$. Contrary to Steele's (1999) conclusions, this implies that rigid rotation cannot account for the evolution of the surface rotational velocity of Be stars.

As frequencies of NRP of Be stars are small in the co-rotating frame (Lee $\&$ Saio 1986, 1987), a correlation between the observed periods and $v \sin i$ would imply weak or no internal differential rotation (Balona 1990, 1995). Fig. 3a shows $v \sin i$ against observed photometric periods of Be stars (Balona 1990, Hubert \& Floquet 1998). According to Sect. 1, we can derive rotational periods from the $v \sin i$ assuming $\omega \gtrsim 0.8$. They are given in Figs. 3b and 3c for $\omega=0.8$ and $\omega=1.0$ against the observed periods. As there is no clear correlation between them, it is possible that Be stars are neither necessarily rigid rotators, nor have rotationally modulated light variations.

Should Be stars be considered differential rotators, knowing that their surface rotation is near critical $(0.8 \lesssim \omega \lesssim 1)$, their total content of rotational kinetic energy must be higher than withstood by critical rigid rotators: energy ratio $\tau_{\mathrm{Be}}=$ kinetic $/$ gravitational $\mid Z \tau_{\text {critical }}^{\text {rigi }} \simeq 0.01$. This may also imply that complete rigid rotation is not attained during the fully convective pre-MS phase.

\section{Evolutionary Status of Be Stars}

The earlier suggestions that Be stars might occur in the secondary contraction phase (Cramping \& Hoyle 1960, Schmidt-Kaler 1964) have been reviewed by Hardorp \& Strittmatter (1970). Assuming that Be stars are critical rotators, 

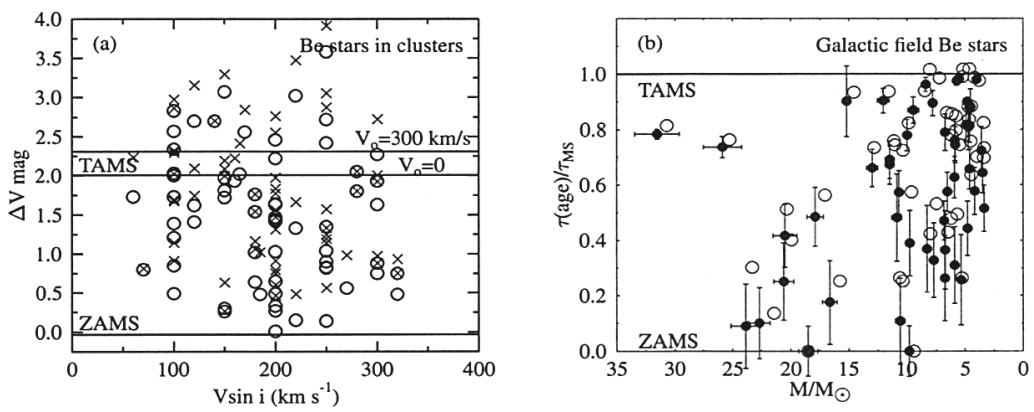

Figure 4. (a) $\Delta V=V-V(\mathrm{ZAMS})$ magnitude differences at constant colours of Be stars in Galactic clusters againt $v \sin i$; (b) $\tau / \tau_{\text {ZAMS }}$ age ratios of Galactic field $\mathrm{Be}$ stars against masses. $\circ$ : data not corrected for rotation; •: data corrected for rotation with uncertainty error bars

they concluded that the Be phenomenon should appear before the TAMS and that these stars are probably fast rotators already in the ZAMS. Another guess at the evolutionary status of Be stars is provided by the frequency distributions of field and cluster Be stars. A detailed study of the fraction of Galactic field Be stars as a function of spectral type and luminosity class was done by Zorec $\&$ Briot (1997). After correcting the stellar countings for several biases, these authors concluded that there is a steady decrease in the number of Be stars from early to late spectral types and that the fractions of Be stars are about the same for dwarfs, subgiants and giants, which indicates that the Be phenomenon may appear at any stage in the MS. The coadded frequency of Be stars in Galactic open clusters against spectral type is the same as for field Be stars (Mermilliod 1982, Feinstein 1990). Several authors have tried to determine at what evolutionary phase Be stars occur by studying clusters, since stellar ages can be easily assessed in this way (cf. Schild \& Romanishin 1976, Mermilliod 1982, Slettebak 1985). The results thus obtained are summarized in Fig. 4a, where $\Delta V=V-V$ (ZAMS) is the difference of magnitudes at constant $(B-V)$ or $(U-B)$ colours. Crosses stand for original data and circles are data corrected for an average $\Delta V$ excess due to the CE (Zorec \& Briot 1991, Wegner 2000). To discover the actual evolutionary displacements, we need still to correct $\Delta V$ for: a) an aspect angle magnitude deviation due to rotational effects (Collins \& Sonneborn 1977, Collins et al. 1991); b) $\Delta V$ displacements that depend on $(B-V)$ or $(U-B)$ changes due, on one hand, to rotational effects and, on the other hand, to colour variations produced by the $\mathrm{CE}$ which introduces positive or negative excesses, depending on the colour index and on whether they concern genuine Be phases or Be-shell phases (Moujtahid et al. 1998). Since the sum of all these effects can exceed $1 \mathrm{mag}$ in $\Delta V$, it is almost impossible to conclude from Fig. 4a at what evolutionary phase Be may occur. In a recent study Fabregat \& Torrejón (2000) have reviewed the dependence of the frequency of Be stars on spectral type and ages of clusters and concluded that the Be phenomenon appears in the second half of their MS lifetime, roughly when there is a sudden increase of the helium and nitrogen abundance in $\mathrm{O}$ and $\mathrm{B}$ stars without emission (Lyubimkov et al. 1997). It should be noted, however, that cluster ages are quite uncertain and that the detection of cluster Be stars is frequently limited to stars with strong emission and/or to the brightest ones. On the other hand, the 

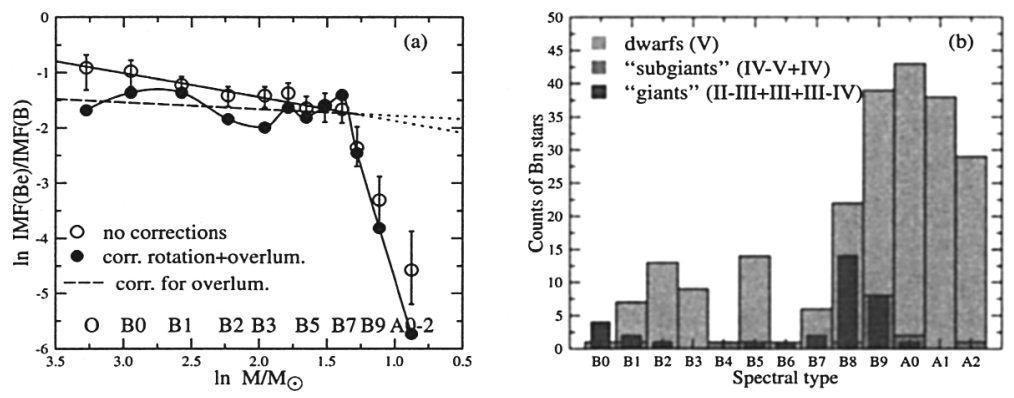

Figure 5. (a) Ratio of Be to B IMF functions against mass; (b) Counts of Bn stars with $V \lesssim 7$ mag against spectral type for different luminosity classes

chemical abundance determination in early-type Be stars shows that while the $\mathrm{C} / \mathrm{N}$ ratio is actually lower than in slowly rotating $\mathrm{B}$ stars, there is no evidence for a change of the He abundance (Frémat et al. 2004). Using a well observed sample of Galactic field Be stars in the BCD system (this volume, p. 81), we have estimated the stellar ages using first only row $\left(\lambda_{1}, D\right)$ parameters, which are already free from $\mathrm{CE}$ perturbations and then, correcting them for rotational effects with $\omega=0.8$. The results are presented in Fig $4 \mathrm{~b}$ which show that the Be phenomenon can be present throughout the MS life span, but the density of points is higher in the upper half of the MS. In Fig $4 \mathrm{~b}$ there is also a tendency for ratios $\tau($ age $) / \tau_{M S}\left(\tau_{M S}=\right.$ time spent by a star in the MS) to be larger for masses $M \lesssim 10 \mathrm{M}_{\odot}$. This could suggest that in low mass stars the Be phenomenon appears later than in the more massive ones, while in the massive stars, due perhaps to the increase of their luminosity during the MS evolution above some $\log L / \mathrm{L}_{\odot} \simeq 4.7$ (Vardya 1985), winds are so strong that the CEs are blown away (Massa 1975).

\section{Nature of the Central Objects in Be Stars}

\subsection{Single Stars}

Using models of rigidly rotating stars, Endal (1982) has shown that the minimum ratio $\eta=$ centrifugal acceleration/gravity, required to reach critical rotation prior to hydrogen exhaustion in the core, is an initial $\eta_{\text {ZAMS }} \gtrsim 0.5$, which would imply $\omega_{\text {ZAMS }} \gtrsim 0.88$ ! According to predictions, based on detailed calculations of the internal angular momentum redistribution (Heger \& Langer 2000, Meynet \& Maeder 2000), a slightly lower $\omega_{\text {ZAMS }}$ is needed in models with low mass-loss $\left(M \lesssim \mathrm{M}_{\odot}\right)$ to recover $\omega \lesssim 1$ from core contraction during the MS evolution, after the initial sudden drop of $\omega$ in the stellar surface. From these results it is expected that initial stellar formation conditions must exist which favor the formation of stars already rotating fast in the ZAMS. They can introduce distinctive characteristics in the initial mass function (IMF), in particular in the IMF of stars "bearing" the Be phenomenon. Fig. 5a reproduces the ratio $\mathrm{IMF}(\mathrm{Be}) / \mathrm{IMF}(\mathrm{B})$ determined for stars earlier than B7 (this volume, p. 87), where a difference between the IMF of B and Be stars seems to be clearly present. This 
figure also shows the extension of the IMF ratios up to the early A-type stars. The apparent lack of Be stars of spectral types later than B7 can be understood in terms of the low ionization state of the $\mathrm{CE}$, which prevents line emission being strong enough for easy detection. However, the number of $\mathrm{Be}$ stars needed to recover a "regular" IMF(Be)/IMF(B) relation over the whole B0-A2 region is, within the errors of stellar samplings limited to the apparent magnitude $V=7$ mag, the same as the number of Bn stars found in the Bright Stars Catalogue and Supplement. Bn stars rotate nearly as fast as Be stars and $80 \%$ of them are dwarfs with maximum occurrence at spectral types B8-A2 (Fig. 5b). From Sects. 1 and 3 it is apparent that Be stars of late spectral types seem to need not only higher values of $\omega$, but also larger age ratios $\tau / \tau_{M S}$ to display the $\mathrm{Be}$ phenomenon. We are tempted then to suggest that either Bn stars have some incipient emission that still needs to be revealed in order to be considered $\mathrm{Be}$ stars, or that they may represent a pre-Be phase.

Maeder et al. (1999) have proposed that the formation of fast rotators can be favored in media with low metallicity, where early decoupling phenomena of magnetic fields could occur. Their suggestion is based on the observation that on average the frequency of Be stars in clusters of nearly the same age steadily increases as the average metallicity decreases from the inner towards the outer regions of our galaxy, up to both Magellanic Clouds (MC). However, the low metallicity may not perhaps be the sole intervening factor to produce fast rotators and/or Be stars, because: a) within a radius of $500 \mathrm{pc}$ near the Sun, the average rotation of B0-B4 type stars goes in the oposite direction to the above global tendency: it decreases from the inner to the outer regions of the Galaxy (Burki \& Maeder 1977, Wolf et al. 1982); b) the frequency of field Be stars in the MC is about the same as that of Galactic field Be stars (Keller et al. 1999); c) due to discrepancies in the estimates according to authors and methods, the fractions of Be stars earlier than B4 in the most populous clusters in Be stars in the Galaxy and in the MC (average age $21 \pm 7 \mathrm{Myr}$ ), do not reveal strong dependence on metallicity: (SMC) 48\% NGC 330 (Grebel 1997, Keller et al. 1999); (LMC) 33\% NGC 2100, 27\% NGC 1818 (Keller et al. 1999); (Galaxy) 36\% NGC 7419 (Pigulski \& Kopacki 2000); 31\% NGC 663 (Pigulski et al. 2001); 36\% NGC 3766 (Shobbrook 1987); 25-50\% NGC 869/884 (Waelkens et al. 1990).

Finally, Wolf et al. (1982) argued that gravitational encounters may produce fast rotators. Since these stars probably behave as runaway stars, not only their number is probably low, but it is difficult to disentangle which of them are or are not survivals from supernova explosions in binary systems (Rinehart 2000, Berger \& Gies 2001).

\subsection{Be Stars in Binaries}

Hitherto we have considered that the Be phenomenon is an intrinsic property of single stars. Binarity among Be stars, at least for periods $P \gtrsim 10 \mathrm{yr}$, is however as frequent as among B stars without emission (Abt 1987). Several authors have proposed that the Be phenomenon might be the consequence of interactions in binary systems:

1) Křiž \& Harmanec (1975) and Harmanec (1987) argued that the Be phenomenon could be the result of an accretion disc formed by Roche-lobe outflowing matter. As the mass transfer is a short-lasting phenomenon, very few $\mathrm{Be}$ 
stars could be explained in this way (Gies 2000, Tarasov 2000). Also, the search for cool companions in many bright Be stars gave negative results (Baade 1992).

2) Packet (1981) predicted that by mass and angular momentum transfer, the mass gainer component can become a rejuvenated and spun up B star. However, it has not yet been shown how these fast rotators can become $\mathrm{Be}$ stars. According to the authors and approximations used, only $50 \%$ of Be stars could be explained by case B of mass transfer in binaries with $P \lesssim 10$ yr (Pols et al. 1991), but perhaps $20 \%$ down to only $5 \%$ (Van Bever \& Vanbeveren 1997). $\mathrm{Be} / \mathrm{X}$-ray stars, where a neutron star orbits an early-type Be stars, are however post-mass-transfer systems (Coe 2000). Theory also predicts a population of $\mathrm{Be}+\mathrm{He}$ star systems, but only one has been so far detected (Gies 2000), and of $\mathrm{Be}+$ white dwarf systems, which up to now escaped detection (Meurs et al. 1992). However, new spectroscopic methods are identifying potential $\mathrm{Be}+\mathrm{sdO}$ and Be+WD candidates today (Maintz et al. 2004). Moreover, in systems where mass-transfer can take place $(P \lesssim 10 \mathrm{yr})$, there is a paucity of $\mathrm{Be}+\mathrm{B}$ systems which must exist if the Be phenomenon is an intrinsic property of stars (Gies 2000). Apart from Algols, ie. Be stars with bright and evolved late type components, there are many iconic Be stars which are either spectroscopic binaries or their binarity is strongly suspected (Gies 2000).

3) Harmanec et al. (2003) have explored the effect of the external force field in a detached binary system on a component at asynchronous and critical rotation and found that a CE can be produced by matter spilled through an inner Lagrangian poịnt, without implying further mass transfer between the components.

It appears then that only a small fraction of stars might do owe their $\mathrm{Be}$ phenomenon to mass transfer in close binaries. In detached binaries, even the onset of the $\mathrm{CE}$ formation might be favored by the external force field, innate fast rotating stars or spun up stellar surfaces (near critical) during the MS evolution seem necessary to produce the Be phenomenon.

Acknowledgements: I am gratful to Dr. A.M. Hubert, Y. Frémat and F. Royer for many and fruitful discussions

\section{References}

Abt, H.A. 1987, IAU Coll. No. 92, p. 470

Baade, D. 1992, IAU Symp. No. 151, (eds.) Kondo et al., p. 147

Balona, L.A. 1975, MNRAS, 173, 449

Balona, L.A. 1990, MNRAS, 245, 92

Balona, L.A. 1995, MNRAS, 277, 1547

Balona, L.A., Henrichs, H.F., \& Le Contel, J.M. 1994, In: Pulsation, Rotation and Mass Loss in Early-Type Stars, IAU Symp. No. 162, (eds.) L.A. Balona, H.M. Henrichs, J.F. Le Contel, Kluwer Acad. Publ.

Berger, D.H., \& Gies, D.R. 2001, ApJ, 555, 364

Briot, D. 1986, A\&A, 163, 67

Burki, G., \& Maeder, A. 1977, A\&A, 57, 401

Chauville, J., Zorec, J., Ballereau, D. et al. 2001, A\&A, 378, 861

Clement, M. 1974, ApJ, 194, 709 
Coe, M.J. et al. 2000, IAU Coll. No. 175, ASP Conf. Ser. 214, 656

Cook, K.H. et al. 1995, IAU Coll. No. 155, ASP Conf. Ser. 83, p. 221

Collins II, G.W., \& Sonneborn, G.H. 1977, ApJS 34, 41

Collins II, G.W., \& Truax, R.J. 1995, ApJ, 439, 860

Collins II, G.W., Truax, R.J., \& Cranmer, S.R. 1991, ApJS, 77, 541

Cramping, J., \& Hoyle, F. 1960, MNRAS, 120, 33

Endal, A.S. 1982, IAU Symp. No. 98, p. 299

Endal, A.S., \& Sofia, S. 1979, ApJ, 232, 531

Fabregat, J., \& Torrejón, J.M. 2000, A\&A, 357, 451

Fainstein, A. 1990, Rev. Mex. Aston. Astrof. 21, 373

Fukuda, I. 1982, PASP, 94, 271

Floquet, M., Hubert, A.M., Hirata, R. et al. 2000, A\&A, 362, 1020

Frémat, Y., Zorec, J., Levenhagen, R. et al. 2004, In: Stellar Rotation, IAU Symp. No. 215, (eds.) A. Maeder, \& P. Eenens, ASP Conf. Ser., this volume

Gies, D.R. 2000, IAU Coll. No. 175, ASP Conf. Ser. Vol. 214, p.

Grebel, E.K. 1997, A\&A, 317, 448

Hardorp, J., \& Strittmatter, P.A. 1970, In: Stellar Rotation, (ed.) A. Slettebak, p. 48 Harmanec, P. 1987, IAU Coll. No. 92, p. 339

Harmanec, P., Bisikalo, D.V., Boyarchuk, A.A. et al. 2003, A\&A (in press)

Hanuschik, R.W., Dachs, J., Baudzus, M. et al. 1993, A\&A, 274, 356

Heger, A., \& Langer, N. 2000, ApJ, 544, 1016

Howarth, I. 2004, In: Stellar Rotation, IAU Symp. 215, (eds.) A. Maeder, \& P. Eenens, ASP Conf. Ser., this volume

Hubert, A.M., \& Hubert, H. 1979, An Atlas of Be Stars, Paris-Meudon Observatory

Hubert, A.M., Floquet, M. 1998, A\&A, 335, 565

Hubert, A.M., Floquet, M., \& Zorec, J. 2000, IAU Coll. 175, p. 348

Jaschek, M., \& Groth, H.G. 1982, In: Be Stars, IAU Symp. No. 98, (eds.) M. Jaschek, \& H.G. Groth, Reidel Publ. Co, Dordrecht-Holland

Jaschek, M., Slettebak, A., \& Jaschek, C. 1981, Be Stars Newsletter No. 4, p.9

Keller, S.C., Wood, P.R., \& Bessel, M.S. A\&AS, 134, 489

Křiž, S., \& Harmanec, P. 1975, Bull. Astron. Inst. Czech. 26, 65

Lee, U., \& Saio, H. 1986, MNRAS, 221, 365

Lee, U., \& Saio, H. 1987, MNRAS, 225, 643

Lyubimkov, L.S., Rostopchin, S.I., Roche, P. et al. 1997, MNRAS, 286, 549

Maeder, A., Grebel, E., Mermilliod, J.C. 1999, A\&A, 346, 459

Maeder, A., \& Zahn, J.P. 1998, A\&A, 334, 1000

Maintz, M., Rivinius, T., Śtefl, S. et al. 2004, In: Stellar Rotation, IAU Symp. 215, (eds.) A. Maeder, \& P. Eenens, ASP Conf. Ser., this volume

Marlborough, J.M. 2000, IAU Coll. No. 175, ASP Conf. Ser. Vol. 214, p. 743

Massa, D. 1975, PASP, 87, 777

Mennickent, R.E., Pietrzynski, G., Gieren, W. et al. 2002, A\&A, 393, 887

Mermilliod 1982, A\&A, 109, 48

Meynet, G. \& Maeder, A. 2000, A\&A, 361, 101

Moujtahid, A., Zorec, J., \& Hubert, A.M. 1998, A\&AS, 129, 289

Moujtahid, A., Zorec, J., \& Hubert, A.M. 1999, A\&A, 349, 151

Neiner, C. 2002, PhD Thesis, University Luis Pasteur, Strasbourg, France 
Neiner, C., Hubert, A.M., Floquet M. et al. 2002, A\&A, 388, 899

Owocki, S.P. 2004, In: Stellar Rotation, IAU Symp. No 215, (eds.) A. Maeder, \& P. Eenens, ASP Conf. Ser., this volume

Packet, W. 1981, A\&A, 102, 17

Pigulski, A., \& Kopacki, G. 2000, A\&AS, 146, 465

Pigulski, A., Kopacki, G., \& Kołaczkowski, Z. 2001, A\&A, 376, 144

Pols O.R., Coté, J., Waters, L.B.F.M. et al. 1991, A\&A, 241, 419

Rinehart, S.A. 2000, MNRAS, 312, 429

Rivinius, T., Baade, D., Štefl, S. et al. 1998, A\&A, 336, 177

Saio, H. 1994, IAU Symp. No. 162, p. 287

Schaller, G., Schaerer, D., Meynet, G. et al. 1992, A\&AS, 96, 269

Schild, R., \& Romanishin, W. 1976, ApJ, 204, 493

Schmidt-Kaler, T. 1964, Veröf. Astr. Inst. Univ. Bonn, No. 70

Shobbrook, R.R. 1987, MNRAS, 225, 999

Slettebak, A. 1976, In: Be and Shell Stars, IAU Symp. No. 70, (ed.) A. Slettebak, Reidel Publ. Co., Dordrecht-Holland

Slettebak, A. 1982, ApJS, 50,

Slettebak, A. 1985, ApJS, 59, 769

Slettebak, A., \& Snow, T.P. 1987, In: Physics of Be Stars, IAU Coll. No. 92, (eds.) A. Slettebak, \& T.P. Snow, Cambridge Univ. Press

Smith, M.A., Henrichs, H.F., \& Fabregat, J. 2000, In: The Be Phenomenon in EarlyType Stars, IAU Coll. No. 175, (eds.) M.A. Smith, H.F. Henrichs, J. Fabregat, ASP Conf. Ser. Vol. 214

Snow, T.P. 1981, ApJ, 251, 139

Steele, I.A. 1999, A\&A, 343, 237

Stoeckley, T.R. 1968a, MNRAS, 140, 121

Stoeckley, T.R. 1968b, MNRAS, 140, 141

Stoeckley, T.R., Buscombe, W. 1987, MNRAS, 227, 801

Struve, O. 1931, ApJ, 73, 94

Struve, O. 1942, Popular Astronomy, 53, 202

Tarasov, A.E. 2000, IAU Coll. No. 175, ASP Conf. Ser. 214, 644

Tassoul, J.L. 1978, Theory of Rotating Stars, Princeton Univ. Press

Van Bever, J., \& Vanbeveren, D. 1997, A\&A, 322, 116

Vardya, V.S. 1985, ApJ, 299, 255

Waelkens, C., Lampens, P., Heynderickx, D. et al. 1990, A\&AS, 83, 11

Wegner, W. 2000, MNRAS, 319, 771

Wolf, S.C., \& Preston, G.W. 1987, ApJS, 37, 371

Wolf, S.C., Edwards, S., \& Preston, G.W. 1987, ApJ, 252, 322

Yudin, R.V. 2001, A\&A 368, 912

Zahn, J.P. 1992, A\&A, 265, 115

Zorec, J. 1998, In: B[e] Stars, Workshop, (eds.) A.M. Hubert \& C. Jaschek, Ap. Sp. Sc. Lib. Vol. 233, p. 27

Zorec, J., Briot, D. 1991, A\&A, 245, 150

Zorec, J., Briot, D. 1997, A\&A, 318, 443

Zorec, J., Frémat, \& Hubert, A.M. 2000a, IAU Coll. 175, p. 330

Zorec, J., Hubert, A.M., \& Moujtahid, A. 2000b, IAU Coll. 175, p. 448 